\title{
Comparative Analysis of the Therapeutic Effect of Disinfectant and Non-disinfectant on the Separation of the Labia Minora in Infants
}

\author{
Qiqi Chen"1, Guangyan Lin'², Jun Xiong³, Ping Wei ${ }^{3}$ \\ ${ }^{1}$ Department of Orthopedics, Children's Hospital of Chongqing Medical University, Chongqing, China \\ 2International wound stomatostomy nurse, Outpatient Surgery, Children's Hospital of Chongqing Medical University, \\ Ministry of Education Key Laboratory of Child Development and Disorders; National Clinical Research Center for Child \\ Health and Disorders; China International Science and Technology Cooperation base of Child development and Critical \\ Disorders; Children's Hospital of Chongqing Medical University, Chongqing, P.R China. Chongqing Key Laboratory of \\ Pediatrics., China
}

${ }^{3}$ Outpatient Surgery, Children's Hospital affiliated to Chongqing Medical University, Chongqing, China

*Corresponding author: Guangyan Lin, 2300727650@qq.com

\begin{abstract}
Objective: To compare the effects of the use and non-use of a disinfectant on the outcomes of separation of the labia minora in infants. Methods: The patients were randomly divided into an experimental group with 24 cases and a control group with 25 cases. In the control group, $1 \%$ iodophor was used to clean and disinfect the large and small labia and the surrounding skin, while normal saline was used in the experimental group. Other procedures such as the surgery and nursing method were the same in both groups. Results: There were no symptoms of urinary tract infection such as redness of the vulva, swelling, pain, and abnormal urination in the test group and control group after three days of follow-up, and no recurrence was seen at one month of follow-up. There was no statistically significant difference between the two groups $(\mathrm{P}>$ 0.05). Conclusion: Use or non-use of a disinfectant to clean the urethral opening before separation of the labia minora has no significant effect on the outcomes, and does not cause postoperative urinary tract infection symptoms. Moreover, non-use of a disinfectant can prevent local irritation and reduce the economic burden on the patients.
\end{abstract}

Keywords: Labial adhesion; Disinfectant; Non-disinfectant

Publication date: November 2021; Online publication: November 30, 2021

\section{Introduction}

Adhesion of the labia minora is one of the common clinical conditions in women and children. It can be a partial or complete adhesion, and manifests as a translucent membranous tissue covering a part of or the entire urethral opening ${ }^{[1]}$. Studies have shown that the incidence of this condition is $3.3 \%{ }^{[2]}$. The current management options include conservative treatment, topical estrogen therapy, separation under anesthesia, freehand separation, etc. ${ }^{[3]}$ According to the Chinese recommendations for the prevention and treatment of adhesions of the labia minora, mild adhesions refer to adhesions between the upper, middle, or lower labia minora, wherein the vaginal and/or urethral orifices cannot be fully exposed. Severe adhesion refers to the complete adhesion of both sides of the labia minora, forming a membranous adhesion line in the middle, the middle of the membrane or visible holes, and the vaginal and urethral opening cannot be exposed completely. It is suggested that in cases with mild or severe asymptomatic adhesions, the labia should be separated after local cleaning with clean water. For clinical symptoms such as severe adhesions accompanied by abnormal urination (frequent urination, overflow of urine, changes in the urinary flow, 
dysuria, pain during urination) and increased vaginal secretions, previous studies have reported local disinfection with iodophor before separation ${ }^{[4]}$. This study compared and analyzed the efficacy of using disinfectant and non-disinfectant solution before the separation of the labia under local smear anesthesia in children with labial minora adhesion, so as to provide a reference for the treatment of clinical children with labial minora adhesion.

\section{Materials and methods}

\subsection{General information}

The children of a children's hospital in Chongqing from April 26 to June 15, 2020 were selected. The inclusion criteria are as follows: (1) children diagnosed with adhesions of the labia minora; and (2) children and their families who volunteer to participate. The exclusion criteria are as follows: (1) children with confirmed symptoms of urinary tract infection; (2) children with verbal communication problems; (3) children with comorbidities; and (4) children on any other medications. The patients were randomly assigned to the experimental group or the control group.

\subsection{Methods}

The child was placed in the lithotomy position, and appropriately secured to ensure there is no movement. The perineal region was completely exposed. The privacy of the patients was safeguarded. Before separating the labia, lidocaine cream for local anesthesia was applied, followed by a 15-min waiting time to allow the anesthesia to kick in. In the control group, $1 \%$ iodophor was used to clean and disinfect the large and small labia and the surrounding skin, while in the experimental group, normal saline was used. After local cleaning, separation of the labia minora was performed with a flat forceps, starting from the weakest point of adhesion. To ensure homogeneity, two wound specialist nurses with work experience of more than 5 years jointly assisted to complete the procedure. After separation, patients of both groups were asked to follow an identical method for home-based care. The use of phellodendron solution to clean the vulva twice a day at home for three days was advised. Additionally, application of comaria oil to both labia majora and labia minora for 7 days was advised. Personalized education about the daily nursing methods at home was provided to the family members of each patient. Cleaning of the vulva with warm water after each defecation for an additional 2-3 times a day with gentle movements and separating the large and small labia to avoid the recurrence of adhesion was advised.

\subsection{Observation index}

The treatment outcomes between the two groups were compared. The parameters evaluated include whether the size of labia hemorrhage and amount of blood loss during separation (small amount: amount of blood loss soaked gauze area $<1 \mathrm{~cm}^{2}$, medium amount: amount of blood loss soaked gauze area $1 \mathrm{~cm}^{2} \leq \mathrm{x} \leq 3$ $\mathrm{cm}^{2}$, large amount: amount of blood loss soaked gauze area $>3 \mathrm{~cm}^{2}$ ), need for oral anti-inflammatory drugs after separation, symptoms of urinary tract infection such as redness, swelling, pain and abnormal urination of the vulva, exudates from the vulva, frequency of vulval cleaning, recurrence rate one month after separation, etc.

\subsection{Statistical analysis}

SPSS version 20.0 software was used for statistical analysis, and values are provided as mean \pm standard deviation (SD). The frequency data were compared by percentage, and the rate was compared by Chisquare analysis. The comparison of two groups with normal distribution was performed using $t$-test. 


\section{Results}

\subsection{General conditions}

In total, 49 subjects were enrolled, including 24 cases in the experimental group and 25 cases in the control group. The difference in baseline data between the groups was not statistically significant $(\mathrm{P}>0.05)$, as shown in Table 1.

Table 1. Comparison of general data between the control group and the experimental group

\begin{tabular}{|c|c|c|c|c|}
\hline \multirow{2}{*}{ Parameters } & Control group $(\mathbf{n}=\mathbf{2 5})$ & Experimental group $(n=24)$ & \multirow{2}{*}{ T value } & \multirow{2}{*}{ P value } \\
\hline & Mean \pm SD & Mean \pm SD & & \\
\hline Age (months) & $12.61 \pm 7.89$ & $12.42 \pm 8.00$ & 0.11 & 0.91 \\
\hline Weight (kg) & $9.17 \pm 2.24$ & $9.31 \pm 2.97$ & 0.19 & 0.85 \\
\hline \multicolumn{5}{|l|}{ Primary caregiver } \\
\hline Parents & 19 & 18 & \multirow{2}{*}{1.00} & \multirow{2}{*}{0.94} \\
\hline Grandparents & 6 & 6 & & \\
\hline \multicolumn{5}{|c|}{ Education level of primary caregiver } \\
\hline Senior high school and below & 17 & 18 & \multirow{2}{*}{1.00} & \multirow{2}{*}{0.59} \\
\hline Junior college or above & 8 & 6 & & \\
\hline \multicolumn{5}{|l|}{ Degree of adhesion } \\
\hline Mild & 12 & 11 & \multirow{2}{*}{1.00} & \multirow{2}{*}{0.88} \\
\hline Severe & 13 & 13 & & \\
\hline \multicolumn{5}{|l|}{ Presence of secretions } \\
\hline Yes & 8 & 6 & \multirow{2}{*}{1.00} & \multirow{2}{*}{0.59} \\
\hline No & 17 & 18 & & \\
\hline
\end{tabular}

\subsection{Comparison of observation indices between the control and experimental groups during separation}

We compared and analyzed the labia bleeding and the amount of bleeding between the control group and the experimental group. Separation hemorrhage in 11 cases of the control group and 9 cases of experimental group was not statistically significant $(\mathrm{P}>0.05)$, The number of cases with no bleeding, small-amount, medium-amount, and large-amount bleeding in the control group were 14 cases, 9 cases, 2 cases, and 0 case, respectively, while the same parameters in the experimental group were 15 cases, 7 cases, 2 cases, and 0 case, respectively. There were no statistically significance differences in bleeding between the two groups ( $\mathrm{P}>0.05)$, as shown in Table 2.

\subsection{Comparison of observation indices between the control group and the experimental group after separation}

In both groups, no oral anti-inflammatory drugs were necessary after the procedure, and there were no symptoms of urinary tract infection such as redness of the vulva, swelling, pain, and abnormal urination, three days after separation. At the one-month follow-up, there were no cases with recurrence. Comparison of vulvar secretions between the two groups showed that there was 1 case of vulvar secretions in the control group and 5 cases in the experimental group; the difference was not statistically significant $(\mathrm{P}>0.05)$, as shown in Table 3. 


\subsection{Frequency of vulva cleaning between the control group and the experimental group before and after separation}

The vulval cleaning frequency between the control group and the experimental group before and after separation was compared. In the control group, the vulvas were cleaned after each defecation in 14 cases before separation, and in 21 cases after separation. In the experimental group, the corresponding numbers were 9 and 22 cases. The difference in the vulval cleaning frequency between the groups was statistically significant $(\mathrm{P}<0.05)$, as shown in Table 4.

Table 2. Comparison of observation indices between the control group and the experimental group at separation

\begin{tabular}{lcccc}
\hline Parameter & Control group $(\mathbf{n = 2 5})$ & Experimental group $(\mathbf{n = 2 4})$ & T value & P value \\
\hline Any bleeding & 11 & 9 & & \\
$\quad$ Yes & 14 & 15 & 1.00 & 0.64 \\
No & & & & \\
Amount of bleeding & 14 & 15 & & \\
No bleeding & 9 & 7 & & \\
Small-amount bleeding & 2 & 2 & 2.00 & 0.88 \\
Medium-amount bleeding & 0 & 0 & & \\
Large-amount bleeding & & & \\
\hline
\end{tabular}

Table 3. Comparison of vulvar secretions between the control group and the experimental group after separation

\begin{tabular}{lcccc}
\hline Parameter & Control group $(\mathbf{n}=\mathbf{2 5})$ & Experimental group $(\mathbf{n}=\mathbf{2 4})$ & T value & P value \\
\hline Secretions from the vulva & & & & \\
Yes & 1 & 5 & 1.00 & 0.75 \\
No & 24 & 19 & \\
\hline
\end{tabular}

Table 4. Comparison of vulva cleaning frequency before and after separation between the control and experimental groups

\begin{tabular}{lccccc}
\hline & \multicolumn{2}{c}{ Vulva cleaning frequency } & T value & P value \\
\cline { 2 - 4 } & $\begin{array}{c}\text { After each bowel } \\
\text { movement }\end{array}$ & $\mathbf{2 - 3}$ times a day & Once per day & & \\
\hline Control group & 14 & 7 & 4 & 1.00 & 0.00 \\
$\quad$ Before the separation & 21 & 3 & 1 & & \\
$\quad$ After the separation & 9 & 12 & 3 & 1.00 & 0.00 \\
Experimental group & 22 & 2 & 0 & & \\
$\quad$ Before the separation & & & & & \\
After the separation & & &
\end{tabular}




\section{Discussion}

\subsection{The use or non-use of disinfectant before separation of adhesions of the labia minora adhesion}

has no influence on the outcomes

Adhesion of the labia minora in children is closely related to the estrogen level and the daily care methods followed at home ${ }^{[5-6]}$. Table 1 shows that there was no statistically significant difference in the general data between the experimental group and the control group, and Table $\mathbf{2}$ shows that there was no statistically significant difference in separation hemorrhage and blood loss between two groups of children with consistent baseline characteristics. The urethral opening and its surrounding skin are colonized by bacteria, such as Staphylococcus epidermidis, Streptococcus, Enterococcus, Acinetobacter, and Mycoplasma. Thus, the perineal environment is not sterile. The guidelines of the American Society of Infectious Diseases and the British guidelines state that the use of disinfectant to clean the urethral opening in the absence of urinary tract infection is not recommended; however, the use of sterile saline to clean the urethral opening prior to surgery is recommended ${ }^{[7-9]}$. Studies have confirmed that adults or children who use or do not use disinfectant to clean the urethral orifice before induration show no statistically significant difference in the occurrence of urinary tract infection ${ }^{[10-11]}$. In this study, children with labia minora adhesion were randomly divided into the control group and the experimental group. Before the separation, disinfectant was used in the control group while it was not used in the experimental group. Table $\mathbf{3}$ shows that there were no symptoms associated with urinary tract infection in the two groups after surgery, and there were no cases of recurrence one month later. There were exudates in the labia minora of some children after surgery, but the difference was not statistically significant, and this might be related to insufficient frequency of vulval cleaning and poor local hygiene of home care in a small number of children, as shown in Table 4. The results of this study confirmed that the use or non-use of disinfectant to clean the urethral opening before the separation of labia minora adhesion in children had no significant effect on the treatment outcome and would not induce postoperative urinary tract infection symptoms. Moreover, it can prevent irritation due to the disinfectant on the skin, since some patients might be sensitive to it. Additionally, non-use of disinfectant reduces the economic cost for the patients ${ }^{[12]}$.

\subsection{Personalized guidance about home care can effectively improve the therapeutic effect and reduce the recurrence rate}

Local application of anesthesia during separation is one of the commonly used methods for the treatment of labia minora adhesions, in addition to local application of estrogen therapy and conservative treatment [3-4]. The results of the Wejde's study confirmed that the success rate of estrogen treatment for labial adhesion was $35 \%$, and the success rate of artificial separation was $80 \%$, while the recurrence rate after artificial separation was far lower than that after estrogen treatment ${ }^{[13]}$. Topical estrogen therapy has adverse effects such as topical irritation (redness, burning) and vulvar pigmentation, with an incidence of $25 \%{ }^{[14-15]}$. In this study, before the separation of labia minora, topical anesthetic drugs were applied to alleviate the pain and discomfort to the children. At the same time, after separation, the methods of home care for children were fully understood, and one-to-one guidance was provided to the main caregivers of the children about the correct methods of home care. Results in Table 4 show that after personalized guidance, the frequency of home care cleaning after separation in both groups was improved compared to that before separation, and the difference was statistically significant. Moreover, no recurrence was seen in both groups. This is consistent with the report by Watanabe et al., ${ }^{[16]}$ which showed that daily local cleaning after manual separation can remove the adhesions and also prevent their recurrence. Thus, this study confirms that even if the labia were not cleaned with a disinfectant before separation, the effective therapeutic effect could be achieved after home care. 


\section{Summary}

When patients do not have symptoms of urinary tract infection before local application of anesthetic drugs to separate labial adhesion, there was no statistical difference in the symptoms of postoperative infection whether or not disinfectant was used to clean the vulva. Moreover, the non-use of disinfectant can also reduce the risk of sensitivity to the disinfectant and reduce the economic cost for the patients. Thus, this study suggests that the use of non-disinfectant solution to clean the vulva, combined with personalized home guidance, can effectively improve the therapeutic effect and reduce the recurrence rate. The limitation of this study is that it was a single-center study. In the future, the sample size can be increased to conduct multi-center studies to further verify the results.

\section{Disclosure statement}

The authors declare no conflict of interest.

\section{References}

[1] Myers JB, Sorensen CM, Wisner BP, et al., 2006, Betamethasone Cream for the Treatment of PrePubertal Labial Third Factor. J Mesometz Gynecc Gynecol, 19(6): 407-411. DOI: 10.1016/j.jpag.2006.09.005

[2] Gibbon KL, Bewley AP, Salisbury JA, 1999, Labial Fusion in Children: A Presenting Feature of Children's Lichen Sclerosus? J Pediatr Dermatol, 16(5): 388-391. DOI: 10.1046/15251470.1999.00102.J.x

[3] Barbosa Ardila SD, Tristancho Baró AI, Suescún Vargas JM, 2017, Sinequia Vulvar: Revisión de Literatura. Arch Argent Pediatr, 115(6): 597-601. DOI: 10.5546/aap.2017.597

[4] Zhang W, 2016, Suggestions on Prevention and Treatment of Infant and Child Labia Minora. Chinese Journal of General Practitioners, 15(12): 918-920.

[5] Gonzalez D, Anand S, Mendez MD, 2020, Labial Adhesions, in StatPearls [Internet], StatPearls Publishing, Treasure Island (FL).

[6] Huang G-Y, Sun W-X, Ye Y-H, 2013, Cause Analysis and Preventive Measures for Labia Minora in Children. Zhonghua Nursing Education, 10(05): 240-241.

[7] Hooton TM, Bradley SF, Cardenas DD, et at., 2010, Diagnosis, Prevention, and Treatment of AlarmAssociated Tract Infection in Patient: 2009 International Clinical Practice Guidelines from the Infectious Diseases Society of AmericaClin, 50: 625-663.

[8] Lobao MJ, Sousa P, 2017, Hospital-Acquired Delivery Tract Infections: Results of a Cohort Study Performed in an Internal Medicine Department. Acta Med Port, 2017: 24. DOI: 10.20344/AMp.8606

[9] Loveday HP, Wilson JA, Pratt RJ, et al., 2014, UK Department of Health. Epic3: National EvidenceBased Guidelines for Preventing Healthcare-Associated Infections in NHS Hospitals in England. J Hosp Infect, 86 Suppl 1: S1-70. DOI: 10.1016/S0195-6701(13)60012-2

[10] Duzkaya DS, Uysal G, Bozkurt G, et al., 2017, Povidone-0.05\% Chlorhexidine Gluconate, or Water for Periphrine Cleaning Before Indwelling Catheterization in a Pediatric Intensive Care: A Randomized Controlled Trial. J Wound Ostomy Continence Nurs, 44(1): 84-88. DOI: 10.1097/WON.0000000000000280

[11] Farsi, S Oliva M, Davidson R, et al., 2009, Periurethral Cleaning the Prior to Urinary Catheterization in Children: Sterile Water Versus 10\% Povidone - Iodine. Clin Pediatr (Phila), 48(6): 656-660. DOI: $10.1177 / 0009922809332587$ 
[12] Fasugba O, Koerner J, Mitchell BG, et al., 2017, Systematic Review and Meta-Analysis of the Effectiveness of Antiseptic Agents for Metal Cleaning in the Prevention of Disease-Associated Water Tract Infections. J Hosp Logs, 95(3): 233-242. DOI: 10.1016/j.jhin.2016.10.025

[13] Wejde E, Ekmark AN, Stenström P, 2018, Treatment with Oestrogen or Manual Separation for Labial Adhesions - Initial Outcome and Long-Term Follow-Up. BMC Pediatr, 18(1): 104. DOI: 10.1186/s12887-018-1018-x

[14] Knudtzon S, Haugen SE, Myhre AK, 2017, Labial Adhesion - Diagnostics and Treatment. Adhesjon av de små kjønnsleppen - diagnostikk og behandling. Tidsskr Nor Laegeforen. 137(1): 31-35. DOI: 10.4045/tidsskr.16.0101

[15] Mayoglou L, Dulabon L, Martin-Alguacil N, et al., 2009, Success of Treatment Modalities for Labial Fusion: A Retrospective Evaluation of Topical and Surgical Treatments. J Cyberoncology Consults for Gynecol. 22(4): 247-250. DOI: 10.1016/j.jpag.2008.09.003

[16] Watanabe T, Matsubara S, Fujinaga Y, et al., 2010, Manual Separation Followed by Local Cleanliness for Pediatric Labial Adhesion. J Obstet Gynaecol Res, 36(3): 667-670. DOI: 10.1111/j.14470756.2010.01192.x 